only 29133 were recruited (ATBC Cancer Prevention Study Group, 1994).

Continuing our previous research (Vartiainen et al, 1994) and analysing random population samples of Finnish subjects, we prospectively monitored mortality of 18344 men (aged 25-64 years) through the National Death Register for a mean of 14.6 years. There were 91 suicides among 7649 smokers and 53 suicides among 10695 non-smokers. In order to replicate the findings of Partonen $e t$ al, we classified cholesterol into the same three categories. Using the Cox model the relative risks were adjusted for identical variables except for carbohydrate intake. Among smokers the unadjusted risks (with $95 \%$ CIs) of suicide increased from 1.00 to $1.48(0.63-3.47)$, and to $1.80(0.75-4.31)$ with increasing cholesterol level. The relative hazards changed clearly after adjustment for covariates $(1.00,1.38,1.62$, respectively), but remained non-significant. In the report by Partonen et al, the relative risks did not change at all after adjustment for covariates, which we find surprising. We found no association between cholesterol and suicide in non-smokers.

Inconsistent findings between these two large longitudinal studies may have resulted from several confounding effects. First, $75 \%$ of the participants in the ATBC study were treated with alpha-tocopherol alone, beta-carotene alone, or both. It is possible, theoretically, that these antioxidants possess some unknown central nervous system effects. Second, the method of suicide may influence the cholesterol-suicide association. Our own findings implicate that very high serum total cholesterol is associated with the increased risk of violent, but not with non-violent suicide (Tanskanen et al, 2000). Third, it has been suggested that cholesterol is only a surrogate marker of changes in dietary polyunsaturated fatty acids, which have been linked to depression (Hibbeln \& Salem, 1995)- one of the strongest risk factors for suicide. Probably various other factors also confound this controversial relationship.

ATBC Cancer Prevention Study Group (1994) The alpha-tocopherol, beta-carotene lung cancer prevention study: design, methods, participant characteristics, and compliance. Annals of Epidemiology, 4, I-10.

Hibbeln, J. R. \& Salem, N., Jr. (1995) Dietary polyunsaturated fatty acids and depression: when cholesterol does not satisfy. American Journal of Clinical Nutrition, 62, I-9.

Partonen, T., Haukka, J., Virtamo, J., et al (1999) Association of low serum total cholesterol with major depression and suicide. British Journal of Psychiatry, $\mathbf{1 7 5}$, 259-262.

Tanskanen, A., Vartiainen, E., Tuomilehto, J., et al (2000) High serum cholesterol and risk of suicide. American Journal of Psychiatry, in press.

Vartiainen, E., Puska, P., Pekkanen, J., et al (1994) Serum cholesterol concentration and mortality from accidents, suicide, and other violent causes. British Medical Journal, 309, 445-447.

A. Tanskanen Research and Development Unit, Department of Psychiatry, Kuopio University Hospital, PO Box 1777, 702II Kuopio, Finland

J.Tuomilehto National Public Health Institute, Helsinki, Finland

H. Viinamäki Department of Psychiatry,

University of Kuopio, Kuopio, Finland

We read with interest the excellent largescale prospective study reported by Partonen et al (1999). They found that low serum total cholesterol appears to be associated with low mood and suicide. However, others have reported conflicting results (McCallum et al, 1994). Weidner et al (1992) found that patients on a cholesterollowering diet were associated with reductions in depression if they were instructed to increase fish consumption. This implied that differences in the composition of polyunsaturated fatty acids (PUFAs) might explain the conflicting finding. The PUFAs are classified into two main groups: omega3 (or n-3) of which the parent essential fatty acid is alpha-linolenic acid (C18:3n-3), and $n-6$, of which the parent essential acid is linoleic acid (C18:2n-6). Maes et al (1999) found that major depression is associated with: significantly decreased total $\mathrm{n}$ 3 fatty acids; increased monounsaturated fatty acids and $\mathrm{C} 22: 5 \mathrm{n} 3$ proportions and increased C20:4n6/C20:5n3 and C22:5n6/ $\mathrm{C} 22: 6 \mathrm{n} 3$ ratios; lower $\mathrm{C} 22: 4 \mathrm{n} 6, \mathrm{C} 20: 5 \mathrm{n} 3$ and $\mathrm{C} 22: 5 \mathrm{n} 3$ fractions in phospholipids; lower C18:3n3, C20:5n 3 and total $\mathrm{n} 3$ fatty acids, and higher $\mathrm{C} 20: 4 \mathrm{n} 6 / \mathrm{C} 20: 5 \mathrm{n} 3$ and $\mathrm{n} 6 / \mathrm{n} 3$ ratios in cholesteryl esters; and lower serum concentrations of phospholipids and cholesteryl esters. These findings are consistent and have shown well-established positive correlation between depression and coronary artery disease. Many studies have documented evidence of hypothalamicpituitary-adrenocortical axis hyperactivity within medication-free patients with major depression, including hypercortisolaemia (Raadsheer et al, 1994). Hypercortisolaemia can induce hypercholesterolaemia, hypertriglyceridaemia and hypertension. These are well known to be predisposing factors of cardiovascular disease. If low serum cholesterol concentrations were linked to increased depression, it would be difficult to interpret the correlation between depression and coronary artery disease. The relationship between cholesterol and depression may not be specific enough.

Maes, M., Christophe, A., Delanghe, J., et al (1999) Lowered omega-3 polyunsaturated fatty acids in serum phospholipids and cholesteryl esters of depressed patients. Psychiatry Research, 85, 275-291.

McCallum, J., Simons, L., Simons, J., et al (1994) Low serum cholesterol is not associated with depression in the elderly: data from an Australian community study. Australian and New Zealand Journal of Medicine, 24, $56 \mathrm{I}-564$

Partonen, T., Haukka, J., Virtamo, J., et al (1999) Association of low serum total cholesterol with major depression and suicide. British Journal of Psychiatry, 175 $259-262$.

Raadsheer, F. C., Hoogendijk, W. J. G., Stam, F. C., et al (1994) Increased numbers of corticotropin-releasing hormone expressing neurons in the hypothalamic paraventricular nucleus of depressed patients. Neuroendocrinology, 60, 436-444.

Weidner, G., Connor, S. L., Hollis, J. F., et al (1992) Improvements in hostility and depression in relation to dietary change and cholesterol lowering. Annals of Internal Medicine, II7, 820-823.

K.-P. Su, S.-Y. Tsai Department of Psychiatry, Taipei Medical College-Affiliated Wan Fang Hospital, No. III, Hsing-Lung Road Sec. 3, Taipei II6, Taiwan

S.-Y. Huang Graduate Institute of Nutrition and Health Science, Taipei Medical College, Taipei, Taiwan

Authors' reply: We analysed data from the ATBC Cancer Prevention Study, which was a primary prevention trial to test whether alpha-tocopherol and beta-carotene supplements would reduce the incidence of lung and other cancers (ATBC Cancer Prevention Study Group, 1994). Smokers were recruited from the total population of elderly men and assessed for eligibility. A previous diagnosis of cancer, current severe angina with exertion, chronic renal insufficiency, cirrhosis of the liver, alcohol dependence, or a disorder limiting participation in the long-term trial were grounds for exclusion.

We concluded that low serum total cholesterol appeared to be associated with low mood. We also found that low serum total cholesterol predicted, after adjusting for risk factors, the occurrence of conditions indicative of poor outcome, such as hospitalisation owing to major depressive disorder and death from suicide. Findings were similar for violent deaths exclusive of suicide. Trial supplementation had no effect 
on the main outcome measures, as we reported in the original paper, nor did the antioxidant supplementation modify the effect of serum total cholesterol on suicide.

Tanskanen et al (above) report in their letter that the risk of suicide was increased with higher serum total cholesterol levels in random samples of Finnish smokers. We do not have any obvious explanation for these conflicting findings, but study populations were rather dissimilar. Their subjects (aged 25-64 years) were mainly from eastern Finland, whereas our subjects (aged 50-69 years) were from south-western Finland. The results of other cohort studies investigating the association of serum total cholesterol levels with death from suicide have been inconsistent, since there has been no association or the association has been inverse in previous studies. Tanskanen et $a l$, as well as $\mathrm{Su}$ et al (above), raise the possibility of dietary fatty acids affecting the occurrence of depressive disorder, which in turn is one of the strongest risk factors for suicide. Our aim is to analyse, in subsequent studies, the relationships between various dietary factors (fats, carbohydrates, and amino acids), depressed mood and suicide risk.

ATBC Cancer Prevention Study Group (1994) The alpha-tocopherol, beta-carotene lung cancer prevention study: design, methods, participant characteristics, and compliance. Annals of Epidemiology, 4, I-10.

T. Partonen, J. Haukka National Public Health Institute, Department of Mental Health and National Research, Mannerheimintie 166, FIN-00300, Helsinki, Finland

J.Virtamo National Public Health Institute, Department of Nutrition, Helsinki, Finland

J. Lönnqvist National Public Health Institute, Department of Mental Health and Alcohol Research, Helsinki, Finland

\section{Motor responses to transcranial magnetic stimulation in schizophrenia}

We read with interest the paper by Boroojerdi et al (1999). Our group found a shorter latency for motor evoked potentials (MEPs) to transcranial magnetic stimulation (TMS) in unmedicated people with schizophrenia of, on average, $2 \mathrm{~ms}$ compared with age- and gender-matched normal subjects (Puri et al, 1996). In contrast, Boroojerdi et al (1999) reported no such latency difference (in their group of medicated patients) and speculated that the presence of antipsychotic medication may have confounded their results. Indeed, our group has previously reported the effects of such medication on the latency and form of the inhibitory silent periods to TMS (Davey et al, 1997), which is known to occur as a result of activating superficial intracortical inhibitory interneurons, possibly GABAergic (Davey et al, 1994). Boroojerdi et al (1999) found a longer latency of transcallosal inhibition to TMS in a group of medicated patients with schizophrenia but did not include a group of drug-naïve patients. It is clearly important to be able to differentiate between pathophysiological mechanisms resulting from schizophrenia and the actions of antipsychotic medication on the corticospinal system.

Boroojerdi, B., Töpper, R., Foltys, H., et al (1999) Transcallosal inhibition and motor conduction studies in patients with schizophrenia using transcranial magnetic stimulation. British Journal of Psychiatry, 175, 375-379.

Davey, N. J., Romaiguère, P., Maskill, D. W., et al (1994) Suppression of voluntary motor activity revealed using transcranial magnetic stimulation of the motor cortex in man. Journal of Physiology, 477, 223-235.

_ , Puri, B. K., Lewis, H. S., et al (1997) The effects of antipsychotic medication on electromyographic responses to transcranial magnetic stimulation of the motor cortex in schizophrenia. Journal of Neurology, Neurosurgery and Psychiatry, 63, 468-473.

Puri, B. K., Davey, N. J., Ellaway, P. H., et al (1996) An investigation of motor function in schizophrenia using transcranial magnetic stimulation of the motor cortex. British Journal of Psychiatry, 169, 690-695.

N. J. Davey Division of Neuroscience \& Psychological Medicine, Imperial College School of Medicine, Charing Cross Hospital, Fulham Palace Road, London W6 8RF

B. K. Puri MRI Unit, MRC Clinical Sciences Centre, Imperial College School of Medicine, Hammersmith Hospital, London WI2 OHS

\section{Clozapine-induced thrombocytosis}

Clozapine is known to cause blood dyscrasias, typically neutropenia and agranulocytosis. A raised platelet count, with clozapine as the sole implicated agent, had been reported to the Committee on Safety of Medicines in three cases. This is the first to be published.

A middle-aged male with ICD schizophrenia failed to respond to neuroleptic medication (haloperidol $25 \mathrm{mg} /$ day, chlorpromazine $500 \mathrm{mg} /$ day), or olanzapine at a dose of $20 \mathrm{mg} /$ day for six weeks. $\mathrm{He}$ was therefore started on clozapine. He continued to receive droperidol $20 \mathrm{mg} /$ day and zopiclone $7.5 \mathrm{mg}$ nocte.

Fifteen days after commencing clozapine he complained of nausea. His clozapine was increased the next day by $25 \mathrm{mg}$ to $300 \mathrm{mg} /$ day. He complained of arthralgia and became hypotensive (b.p. 90/ $60 \mathrm{mmHg}$ ). Clozapine was stopped and the symptoms subsided over 36 hours. Clozapine was then restarted at a dose of $100 \mathrm{mg}$ twice daily. He re-developed hypotension, arthralgia, malaise and sweating after one dose. He was apyrexial. Five days after the onset of nausea, the platelet count was $454 \times 10^{9} / 1$ (normal range: 150 $\left.450 \times 10^{9} / 1\right)$, the erythrocyte sedimentation rate (ESR) $70 \mathrm{~mm} / \mathrm{h}$ and the C-reactive protein 103. Eight days later the ESR had fallen to $<5 \mathrm{~mm} / \mathrm{h}$ but the platelet count had risen to $774 \times 10^{9} / 1$. Five days later the platelet count had fallen to $393 \times 10^{9} / 1$ and subsequently returned to normal.

Muller et al (1991) reported fever 7-15 days after commencing clozapine in $12 \mathrm{pa}-$ tients with non-specific inflammatory parameters, including a raised white cell count, ESR and C-reactive protein. They did not comment on platelet changes. This case has similar symptoms but without pyrexia. The rapid re-emergence of symptoms on rechallenge suggests an immune response to the drug, and both thrombocytosis and thrombocytopenia are recognised features of such a reaction.

Muller, H., Manns, M., Hammes, E., et al (1991) Studies on inflammatory side effects of clozapine. Biological Psychiatry, 29 (suppl.) 4I5S-4I6S.

M. E. Hampson Rosebery House, Waterford Street, Old Basford, Nottingham NG6 OHG

\section{Paternal age and schizophrenia in dizygotic twins}

Crow (1999) reported that dizygotic twinning increases with parental age as does the incidence of schizophrenia. Our study of 574 patients with schizophrenia showed that the incidence of schizophrenia increases with paternal age (Raschka, 1998). Scientific publications reported increased incidence of at least 12 illnesses with increased paternal age. The rate of mutations in spermatogenesis increases with age (Penrose, 1955; Vogel \& Motulsky, 1979; Raschka, 1995; Sankaranarayanan, 1998). Other age-related changes are also known 